\title{
Ground-state properties of a large Coulomb-blockaded quantum dot
}

\author{
Eugene B. Kolomeisky ${ }^{(1)}$, Michael Timmins ${ }^{(1)}$ and Ryan M. Kalas ${ }^{(2)}$ \\ (1) Department of Physics, University of Virginia, \\ P. O. Box 400714, Charlottesville, Virginia 22904-4714, USA
(2) Department of Physics and Astronomy, Washington State University, Pullmann, Washington, 99164-2814, USA
}

\begin{abstract}
Using renormalization-group techniques we analyze equilibrium properties of a large gated quantum dot coupled via a long and narrow channel to a reservoir of electrons. Treating the electrons in the channel as one-dimensional and interacting, we demonstrate that for nearly-open dot and not very strong spin fluctuations the ground-state properties as a function of the gate voltage are non-analytic at the points of half-integer average dot population. Specifically, the exact result of K. A. Matveev, Phys. Rev. B 51, 1743 (1995), that the dot capacitance shows periodic logarithmic singularities is rederived as a special case corresponding to non-interacting electrons. We demonstrate that this conclusion also holds in the presence of $S U(2)$ spin symmetry, and argue that logarithmic singularities persist as long as the Coulomb blockade is destroyed which will be the case for sufficiently large tunneling and not very strong interparticle repulsions. We show that interparticle repulsions aid the Coulomb blockade to survive disordering effect of zero-point motion provided the tunneling is sufficiently weak. Upon increase of tunneling, the Coulomb blockade disappears through a Kosterlitz-Thouless phase transition characterized by a dot population jump whose magnitude is only determined by interparticle repulsions in the channel. Similar conclusions are applicable to systems without $S U(2)$ spin symmetry except that the logarithmic singularity of the capacitance is replaced by a power-law non-analyticity whose exponent characterizes the degree of spin fluctuations.
\end{abstract}

PACS numbers: 71.10.Pm, 73.43.Jn, 73.21.Hb, 72.10.Fk

\section{INTRODUCTION}

Quantum dots are mesoscopic objects whose properties, like those of ordinary atoms, can vary appreciably upon addition or removal of a single electron 1 . This fact makes them an active playground of studying the manybody physics under the conditions of confinement.

A simple picture of a dot pertinent to understanding its equilibrium properties is that of a container of electrons connected to a lead via a single constriction and placed nearby a gate electrode. The role of the latter is to tune the chemical potential of the dot to host a desirable number of electrons which is generally not integer. On the other hand, the electrons are discrete, and can only hop onto or off the dot one at a time. Therefore they enter or leave the dot only at special values of the gate voltage corresponding to half-integer dot population. This effect well identified by the early 1990's is called the Coulomb blockade ${ }^{2}$. One of its hallmarks is the dependence of the population of the dot on the gate voltage consisting of plateaus corresponding to an integer number of electrons on the dot - the famed Coulomb staircase.

Quantum-mechanical tunneling of charge between the connecting lead and the dot has been argued to smear the classical Coulomb staircase even at zero temperature ${ }^{3}$. This idea is supported by a connection of the quantum dot problem to a Kondo problem $\stackrel{4}{*}$. In the limit of almost zero tunneling the population dynamics on the dot can be approximated by that of a two-level system, with the levels corresponding to population states of the dot differing by unity ${ }^{4}$. Changing the population on the dot is then akin to flipping an impurity spin- $1 / 2$ while the deviation of the average dot population from the half-integer value is analogous to a magnetic field applied to the Kondo spin. For a single-channel Kondo problem pertinent to the spinless version of the quantum dot problem, the spin susceptibility is finite at zero applied field. This implies smearing of the population jumps with variation of the gate voltage.

Complementary treatments of the strong-tunneling regime ${ }^{5.6}$ led to a conjecture that the rounded Coulomb staircase of the weak-tunneling limit smoothly evolves as tunneling is increased into a strictly linear function of the gate voltage.

A spinless version of the Coulomb blockade problem has been recently revisited ${ }^{7}$ where additionally it was assumed that the dot is connected to a one-dimensional reservoir so that interparticle interactions have to be taken into account. It has been demonstrated that as the strength of the tunneling is tuned, changing the dot from open to closed, a modified Coulomb staircase reappears under certain circumstances. This was arrived at by mapping the zero-temperature dynamics of interacting electrons to the classical statistical mechanics of a one-dimensional Ising model with ferromagnetic interactions decaying as the inverse-square of distance. This led to the conclusion that all experimental systems must be either of the Kondo subclass (to which the connection to the Kondo problem ${ }^{4}$ applies) or of a novel tricritical type.

Here we reconsider the problem of the Coulombblockaded quantum dot and take into account both the electron spin and electron-electron interactions in the channel connecting the dot with the electron reservoir. This is relevant because the strongest argument in fa- 
vor of the Kondo analogy has been given for the noninteracting version of this problem: Matveev $\underline{\underline{6}}$ found exactly that in the high-transparency limit, the capacitance of the dot has a logarithmic singularity whenever the average population of the dot is half-integer. The weaktunneling analysis of this problem ${ }^{4}$ which used the solution of the two-channel Kondo problem also found a logarithmic divergence which led to the contention that this singularity of the capacitance persists for arbitrary tunneling. These predictions ${ }^{6}$ found partial experimental support ${ }^{8}$ thus making the issue practically relevant.

The present work investigates the problem in a phenomenological fashion by using renormalization-group techniques which have an advantage of generality, thus complementing earlier calculations. Specifically, we show that the logarithmic singularity of the capacitance ${ }^{4.6}$ is a consequence of the assumption that the electrons in the constriction are non-interacting. We also point out that the same conclusion holds in the presence of $S U(2)$ spin symmetry as long as the Coulomb blockade is destroyed. Moreover, renormalization-group methods allow us to see clearly possible limitations of existing approximations, thus motivating future work.

The organization of this paper is as follows. In Section II we set up the problem in the form of a zerodimensional quantum field theory describing the dynamics of the charge and spin of the dot. For a nearly-open dot (Section III), the population of the dot tracks the gate voltage and only the spin dynamics described by a sine-Gordon like theory is relevant. In Section IIIA this field theory is studied perturbatively in small reflection amplitude. This exercise demonstrates that physically interesting cases are beyond the perturbative approach. Section IIIB contains a heuristic argument which allows us to guess the answers for situations when the perturbation theory fails. These conclusions are placed on a sounder footing in Section IIIC where renormalizationgroup analysis is employed. The novel feature here is a systematic calculation of the renormalization of the ground-state energy which allows, for example, to exactly reproduce the logarithmic divergence of the capacitance ${ }^{6}$. In Section IIID we draw a parallel between these results and those found earlier for the standard sine-Gordon theory.

In Section IV we study the opposite limit of a nearlyclosed dot. For half-integer average dot population the dynamics of electrons hopping onto and off the dot is approximated by that of a two-state system. The zerotemperature properties of the latter can be inferred from the classical statistical mechanics of a two-component model resembling the one-dimensional Ising model with interactions decaying as the inverse-square of distance. An "entropy" argument which in Section IVA is applied to this model provides us with the range of stability of the regime of zero tunneling. These conclusions are refined in Section IVB via a renormalization-group calculation perturbative in tunneling amplitude. This calculation indicates that whenever the tunneling is relevant, the non-analytic behavior of the ground-state properties of the dot has to be captured by the results found for a nearly-open dot. In Section IVC this statement is made more quantitative via a heuristic argument which shows how the weak-tunneling results away from half-integer dot population can be inferred from those of Sections III and IVB. We conclude (Section V) by summarizing our results and emphasizing possible limitation of the two-state approximation of the hopping dynamics for a nearly-closed dot.

\section{STATEMENT OF THE PROBLEM}

Following Matveev ${ }^{\underline{6}}$ we consider a large quantum dot of capacitance $C_{0}$ whose charging energy $E_{C}=e^{2} / 2 C_{0}$ is significantly larger than the distance between the energy levels on the dot. The dot is placed near a gate and connected to a reservoir of spin- $1 / 2$ electrons through a channel which we assume to be so narrow and long that the electrons in the channel are essentially one-dimensional and their interactions have to be taken into consideration. Although there are no known experimental realizations of this model, appropriate experiments might become possible in the future. Additionally, this represents a generalization of the model due to Matveev ${ }^{\underline{6}}$ in a way that adds interactions violating $S U(2)$ spin symmetry because it is interesting from theoretical point of view. The corresponding long-wavelength low-energy Euclidian action has the form

$$
\begin{aligned}
A & =\frac{1}{2} \int \sum_{\nu=c, s} d x d \tau \rho_{\nu}\left(\left(\frac{\partial u_{\nu}}{\partial \tau}\right)^{2}+c_{\nu}^{2}\left(\frac{\partial u_{\nu}}{\partial x}\right)^{2}\right) \\
& +\int d \tau V\left(u_{\uparrow}(0, \tau), u_{\downarrow}(0, \tau)\right)
\end{aligned}
$$

The first harmonic term describes interacting onedimensional electrons inside the channel. Here the displacements of the electrons of each spin population with respect to their classical positions are represented by the fields $u_{\uparrow}(x, \tau)$ and $u_{\downarrow}(x, \tau)$ where arrows indicate the spin direction, $x$ is the one-dimensional coordinate along the channel and $\tau$ is the imaginary time. Written in terms of the center of mass ("charge") and reduced relative displacement ("spin") fields

$$
u_{c}=\frac{u_{\uparrow}+u_{\downarrow}}{2}, \quad u_{s}=\frac{u_{\uparrow}-u_{\downarrow}}{2},
$$

correspondingly, the first term of (1) is diagonal. The parameters $\rho_{\nu}$ and $c_{\nu}$ are the macroscopic mass densities and sound velocities in the charge and spin sectors ${ }^{9}$.

The harmonic (Luttinger) liquid exponents

$$
g_{\nu}=\frac{\pi \hbar(2 n)^{2}}{\rho_{\nu} c_{\nu}}
$$

characterize interparticle interactions in the charge $(\nu=$ $c)$ and $\operatorname{spin}(\nu=s)$ channels 9 . They are formed out of 
the parameters of the harmonic part of the action (1) and the one-dimensional electron density $n$ within each spin population. Although non-interacting electrons correspond to $g_{c, s}=2$, in what follows we will consider more general values of the exponents.

We also note that either spin population creates a periodic potential for its opposite spin counterpart which may cause spin ordering. For weak short-range interaction between the electrons of opposite spin this can be modeled by adding to (11) a term proportional to $\int d x d \tau \cos 4 \pi n u_{s}$. We however assume that such interactions are irrelevant in the renormalization-group sense $\mathrm{e}^{10}$, and thus the spin ordering does not take place. This would be the case, for example, in the presence of $S U(2)$ spin symmetry (the continuum Hubbard model with repulsive interactions can serve as an example) where $g_{s}=2$. More generally we will require that the degree of quantum fluctuations in the spin channel is sufficiently large $\mathrm{e}^{-}$, specifically $g_{s} \geq 2$.

The second term of the action (1) is due to the tunneling and Coulomb repulsion on the dot and the arguments of the potential $V$ are evaluated at $x=0$ which can be taken to be the narrowmost place of the channel ${ }^{6}$.

Since the interactions in (1) are restricted to a point, the harmonic degrees of freedom away from $x=0$ can be integrated out. This is equivalent to minimizing the action (1) with fixed values of the fields $u_{c, s}$ at the origin followed by the substitution of the solution back into (1) and evaluation of the outcome. The conditions $\delta A / \delta u_{c, s}$ $=0$ reduce to the Laplace equations

$$
\frac{\partial^{2} u_{c, s}}{\partial \tau^{2}}+c_{c, s}^{2} \frac{\partial^{2} u_{c, s}}{\partial x^{2}}=0
$$

whose solutions satisfying the boundary conditions $u_{c, s}(0, \tau) \equiv u_{c, s}(\tau)$ have the form

$$
u_{c, s}(x, \tau)=\int_{-\infty}^{+\infty} \frac{d \omega}{2 \pi} u_{c, s}(\omega) \exp \left(i \omega \tau-|\omega||x| / c_{c, s}\right)
$$

where we introduced the Fourier transforms $u_{c, s}(\omega)=$ $\int d \tau u_{c, s}(\tau) \exp (-i \omega \tau)$. Substituting (15) in (11), performing the integration, and introducing two new fields $\theta_{c}$ and $\theta_{s} / 2$ corresponding to the fluctuating electron number and spin on the dot, respectively

$$
\theta_{c}=n\left(u_{\uparrow}+u_{\downarrow}\right)=2 n u_{c}, \quad \theta_{s}=n\left(u_{\uparrow}-u_{\downarrow}\right)=2 n u_{s}
$$

we find the effective action

$$
A_{e f f}=\pi \hbar \sum_{\nu=c, s} \int_{|\omega| \leq \Lambda_{0}} \frac{d \omega}{2 \pi g_{\nu}}|\omega|\left|\theta_{\nu}(\omega)\right|^{2}+\int d \tau V\left(\theta_{c}, \theta_{s}\right)
$$

where $\Lambda_{0}$ is the high-frequency cutoff. Here without the loss of generality we restrict ourselves to the sharp cutoff model with all the $|\omega|>\Lambda_{0}$ modes eliminated.

For the problem at hand the potential $V$ entering the actions (11) and (7) is a sum of two contributions:

$$
V=E_{C}\left(\theta_{c}-N\right)^{2}+U\left(u_{\uparrow}, u_{\downarrow}\right) \equiv E_{C}\left(\theta_{c}-N\right)^{2}+U\left(\theta_{c}, \theta_{s}\right)
$$

where the first term is the charging energy with dimensionless parameter $N$ proportional to the gate voltage $e^{6}$. The second, tunneling term $U\left(u_{\uparrow}, u_{\downarrow}\right)$ is periodic in both arguments with period $n^{-1}$ which reflects the fact that as either of the spin populations moves along the channel it experiences a periodic potential with the period set by interparticle spacing. Equivalently, it is a manifestation of the discreteness of the electrons - the dot "prefers" to host an integer number of electrons thus implying that the function $U\left(u_{\uparrow}, u_{\downarrow}\right)$ has minima at integer values of $n u_{\uparrow, \downarrow}$. The classical limit of "closed" dot is recovered by increasing the amplitude of $U$ to infinity while the regime of perfect transmission is reached by decreasing the amplitude of $U$ to zero. For example, for the reflection amplitude satisfying the condition $|r| \ll 1$, the form

$$
\begin{aligned}
U & =-\frac{D|r|}{\pi}\left(\cos 2 \pi n u_{\uparrow}+\cos 2 \pi n u_{\downarrow}\right) \\
& =-\frac{2 \gamma \Lambda_{0} \hbar|r|}{\pi} \cos \pi \theta_{c} \cos \pi \theta_{s}
\end{aligned}
$$

was derived earlier ${ }^{6}$. Here $D$ is the high-energy cutoff (bandwidth) used by Matveev $\underline{\underline{6}}$ and $\gamma=e^{\mathbf{C}}$ with $\mathbf{C} \approx$ 0.5772 being Euler's constant. Shortly it will become clear why the energy and frequency cutoffs in (9) are related by $D=\hbar \gamma \Lambda_{0}$.

We note that without the charging term, $E_{C}=0$, Eqs.(7) and (8) describe the dynamics of spin- $1 / 2$ electrons in the presence of a localized inhomogeneity 11 . Additionally, for the special case $g_{c}=g_{s}$ these equations capture the physics of resonant tunneling of interacting spinless electrons onto and off a dot formed by two tunneling barriers in the presence of a gate which couples to the electrons between the barriers 11 .

\section{NEARLY-OPEN DOT}

Combining Eq.(17), (8), and (9) we arrive at the effective action describing the physics of nearly-open dot

$$
\begin{gathered}
A_{\text {eff }}=\pi \hbar \sum_{\nu=c, s} \int_{|\omega| \leq \Lambda_{0}} \frac{d \omega}{2 \pi g_{\nu}}|\omega|\left|\theta_{\nu}(\omega)\right|^{2} \\
+\int d \tau\left(E_{C}\left(\theta_{c}-N\right)^{2}-\frac{2 \gamma \Lambda_{0} \hbar|r|}{\pi} \cos \pi \theta_{c} \cos \pi \theta_{s}\right)
\end{gathered}
$$

For almost perfect transmission $|r| \ll 1$ the action (10) is minimized for $\theta_{c}=N$, and the electron number field $\theta_{c}$ can be eliminated. This is done by writing $\theta_{c}=N+\delta \theta_{c}$ where $\delta \theta_{c}$ is a fluctuation and averaging over the unperturbed, $|r|=0$, ground state, i. e. over the Gaussian part of (10) involving the fluctuating number field $\theta_{c}$ :

$$
A_{0 c}=\int_{|\omega| \leq \Lambda_{0}} \frac{d \omega}{2 \pi}\left(\frac{\pi \hbar}{g_{c}}|\omega|+E_{c}\right)\left|\theta_{c}(\omega)\right|^{2}
$$


This can be used to calculate the mean-square fluctuation of the number of electrons on the dot

$$
<\left(\delta \theta_{c}\right)^{2}>_{0 c}=\frac{g_{c}}{2 \pi^{2}} \ln \left(1+\frac{\pi \hbar \Lambda_{0}}{E_{C} g_{c}}\right)
$$

where $\left\langle>_{0 c}\right.$ denotes an expectation value computed with $A_{0 c}$, Eq. (11). Similar to the spinless case ${ }^{6}$, the fluctuation (12) is finite due to the presence of the Coulomb repulsion on the dot. We note however that for the special case of free electrons, $g_{c}=2$, Eq.(12) agrees with previous conclusion 6 only with logarithmic accuracy. When the frequency cutoff $\Lambda_{0}$ vanishes or the charging energy $E_{C}$ diverges, the fluctuation (12) vanishes. Additionally, when $E_{C}=0$, the fluctuation (12) diverges. These conclusions are in accordance with the physical expectation.

The result (12) allows us to find the expectation value

$$
<\cos \pi \theta_{c}>_{0 c}=\left(1+\frac{\pi \hbar \Lambda_{0}}{E_{C} g_{c}}\right)^{-g_{c} / 4} \cos \pi N
$$

With this in mind the action (10) simplifies to a reduced form involving only the spin field $\theta_{s}$

$$
\frac{A_{s}}{\hbar}=\pi \int_{|\omega| \leq \Lambda_{0}} \frac{d \omega}{2 \pi g_{s}}|\omega|\left|\theta_{s}(\omega)\right|^{2}-v_{0} \Lambda_{0} \int d \tau \cos \pi \theta_{s}
$$

where the dimensionless parameter

$$
v_{0}=\frac{2 \gamma|r|}{\pi}\left(1+\frac{\pi \hbar \Lambda_{0}}{E_{C} g_{c}}\right)^{-g_{c} / 4} \cos \pi N
$$

accumulates salient information about the problem at hand, and the parameter $N$ has a meaning of the average population of the dot.

\section{A. Perturbation theory}

We start by computing a correction to the ground-state energy of the dot $\delta E$ perturbative in the interacting part of the action (14)

$$
\frac{A_{i n t}}{\hbar}=-v_{0} \Lambda_{0} \int d \tau \cos \pi \theta_{s}
$$

A quick way to arrive at corresponding expression is to notice that the action (14) can be formally recognized as a Hamiltonian of a one-dimensional classical statistical mechanics problem with the imaginary time coordinate $\tau$ corresponding to fictitious space direction ${ }^{12,13}$. Then the zero-point motion is analogous to thermal fluctuations, and the result can be inferred from the formulas of thermodynamic perturbation theory ${ }^{14}$. Restricting ourselves to second order we have

$$
L \delta E=<A_{\text {int }}>_{0 s}-\frac{1}{2 \hbar}<\left(A_{\text {int }}-<A_{\text {int }}>_{0 s}\right)^{2}>_{0 s}
$$

Here $L=\hbar / T$ is the size of the fictitious classical system in the imaginary time direction and $T$ is the temperature; the zero-temperature limit is equivalent to the $L \rightarrow \infty$ limit. The notation $\left\langle>_{0 s}\right.$ indicates an expectation value computed with the harmonic, $v_{0}=0$, part of $A_{s}$, Eq.(14):

$$
\frac{A_{0 s}}{\hbar}=\pi \int_{|\omega| \leq \Lambda_{0}} \frac{d \omega}{2 \pi g_{s}}|\omega|\left|\theta_{s}(\omega)\right|^{2}
$$

In order to compute the first-order correction, $\delta E_{1}=<$ $A_{\text {int }}>_{0 s} / L$, we need to know the mean-square fluctuation of the spin field $\theta_{s}$ which can be found to be

$$
<\theta_{s}^{2}>_{0 s}=\frac{g_{s}}{2 \pi} \int_{|\omega| \leq \Lambda_{0}} \frac{d \omega}{2 \pi|\omega|}=\frac{g_{s}}{2 \pi^{2}} \ln \left(\Lambda_{0} L\right)
$$

This implies that

$$
L \delta E_{1}=<A_{\text {int }}>_{0 s}=-v_{0} \hbar\left(\Lambda_{0} L\right)^{1-g_{s} / 4},
$$

i.e. the first-order correction to the ground-state energy $\delta E_{1} \propto L^{-g_{s} / 4}$ vanishes in the zero-temperature limit. Similar to the free-fermion case ${ }^{6}, g_{c, s}=2$, this is a consequence of divergent spin fluctuations (19).

In order to calculate the second-order correction $\delta E_{2}=$ $-<\left(A_{\text {int }}-<A_{\text {int }}>_{0 s}\right)^{2}>_{0 s} / 2 L \hbar$, we will need the correlation function

$$
\begin{aligned}
& \chi(\tau)=<\left(\theta_{s}(\tau)-\theta_{s}(0)\right)^{2}>_{0 s} \\
& =\frac{g_{s}}{\pi} \int_{|\omega| \leq \Lambda_{0}} \frac{d \omega}{2 \pi} \frac{1-\cos \omega \tau}{|\omega|}=\frac{g_{s}}{\pi^{2}} \operatorname{Cin}\left(\Lambda_{0}|\tau|\right)
\end{aligned}
$$

where $\operatorname{Cin}(t)$ is a cosine integral ${ }^{15}$. As $t \rightarrow 0$ the function $\operatorname{Cin}(t)$ vanishes as $t^{2} / 4$ while as $t \rightarrow \infty$, it diverges logarithmically, $\mathrm{Cin}(t) \rightarrow \ln (\gamma t)$. The latter asymptotics also implies the large-argument logarithmic divergence of the correlation function (21), $\chi(\tau)=\left(g_{s} / \pi^{2}\right) \ln \left(\gamma \Lambda_{0}|\tau|\right)$. For noninteracting electrons, $g_{s}=2$, this can be compared with the result implicit in Eq.(34) of Matveev ${ }^{6}$ : $\chi(\tau)=\left(2 / \pi^{2}\right) \ln (D|\tau| / \hbar)$. Therefore the energy and the frequency cutoffs, $D$ and $\Lambda_{0}$, respectively, are related by $D=\hbar \gamma \Lambda_{0}$, which was already employed in Eq.(9).

The expression for the second-order correction $\delta E_{2}$ can be simplified to

$\delta E_{2}=-\frac{\left(v_{0} \hbar \Lambda_{0}\right)^{2}}{2 \hbar} \int_{0}^{\infty} d \tau \exp \left(-\frac{\pi^{2} \chi(\tau)}{2}\right)=-\frac{v_{0}^{2} \hbar \Lambda_{0}}{2} B\left(g_{s}\right)$,

where the function

$$
B\left(g_{s}\right)=\int_{0}^{\infty} d t \exp \left(-\frac{g_{s}}{2} \operatorname{Cin}(t)\right)
$$

is only defined for $g_{s}>2$. The evaluation of the integral in Eq.(23) employing the logarithmic approximation of the cosine integral, $\operatorname{Cin}\left(t \leq \gamma^{-1}\right)=0$ and $\operatorname{Cin}\left(t>\gamma^{-1}\right)=$ $\ln (\gamma t)$, gives

$$
B\left(g_{s}\right) \approx \frac{g_{s}}{\gamma\left(g_{s}-2\right)}
$$


Although this estimate becomes asymptotically exact as $g_{s} \rightarrow 2$ from above, as $g_{s}$ increases, its accuracy degrades. Specifically, as $g_{s} \rightarrow \infty$, the integral (23) is dominated by the region of small $t$ where the logarithmic approximation is not adequate and we find instead $B\left(g_{x}\right) \approx\left(2 \pi / g_{s}\right)^{1 / 2}$. However exceedingly large values of the spin correlation exponent $g_{s}$ are non-physical and will not be considered.

In order to evaluate a low-temperature contribution into $\delta E_{2}$ we need to replace the upper integration limit in Eq.(23) with $\Lambda_{0} L / 2 \gg 1$. As a result we find

$$
\begin{aligned}
\delta E_{2} & =-\frac{v_{0}^{2} \hbar \Lambda_{0}}{2} B\left(g_{s}\right)\left(1-\frac{2\left(\gamma \Lambda_{0} L / 2\right)^{1-g_{s} / 2}}{\gamma\left(g_{s}-2\right) B\left(g_{s}\right)}\right) \\
& \approx-\frac{v_{0}^{2} \hbar \Lambda_{0}}{2} B\left(g_{s}\right)\left(1-\frac{2\left(\gamma \Lambda_{0} L / 2\right)^{1-g_{s} / 2}}{g_{s}}\right)
\end{aligned}
$$

where in the last step we used the approximation (24). For $g_{s}>2$ we may take the limit of zero temperature, $L \rightarrow \infty$, which takes us back to Eq.(22), while in the presence of $S U(2)$ spin symmetry, $g_{s}=2$, we find with logarithmic accuracy

$$
\delta E_{2}=-\frac{v_{0}^{2} \hbar \Lambda_{0}}{2 \gamma} \ln \left(\Lambda_{0} L\right)
$$

In the zero-temperature limit, $L \rightarrow \infty$, this result diverges which means that a non-perturbative calculation is required. The logarithmic divergence (26) is analogous to its free-fermion counterpart $g_{c, s}=2$ found earlier ${ }^{6}$.

Additionally, the special place of the limit of noninteracting electrons can be seen by taking the continuum limit, $\Lambda_{0} \rightarrow \infty$, in Eq. (26). This only leaves the logarithmic dependence on the microscopic cutoff, $\delta E_{2}=-\left(4 \gamma|r|^{2} E_{C} / \pi^{3}\right) \ln \left(\Lambda_{0} L\right)$, thus implying that the prefactor is solely determined by macroscopic quantities.

\section{B. Beyond perturbation theory: heuristic argument}

In order to go beyond the perturbation theory, first we need to clarify its range of applicability. A naive expectation that the perturbative result (22) is reliable for $g_{s}>2$ as long as $\left|v_{0}\right| \ll 1$ is misleading. To find the correct condition we adopt an argument due to J. Lajzerowicz originally given in the classical context of the roughening phase transition ${ }^{16}$ :

The cumulant expansion (17) is performed in powers of $A_{\text {int }} / \hbar$ which implies that the perturbation theory is applicable as long as $\left|<A_{\text {int }}>_{0 s} / \hbar\right| \ll 1$. Inspection of Eq.(20) shows that this will be the case only for $g_{s} \geq 4$ and $\left|v_{0}\right| \ll 1$. One can also arrive at the same conclusion by applying the standard argument ${ }^{14}$ which compares the magnitudes of the first and second-order terms of the expansion (17).

The physics in the non-perturbative regime $2 \leq g_{s}<4$ can be understood heuristically by noticing that the perturbative treatment assumes that spin fluctuations grow with scale without bound (see Eq.(19) ) which is indeed the case for $g_{s} \geq 4$. This assumption, however, breaks down for $g_{s}<4$ because spin fluctuations are suppressed at scales exceeding a characteristic value $L_{c}$ which has a meaning of the correlation length of the fictitious classical statistical mechanics problem corresponding to the action (14). This scale marks the verge of applicability of the perturbation theory, $\left|<A_{\text {int }} / \hbar\right| \simeq 1$, which with the help of Eq.(20) leads to the estimate

$$
L_{c} \simeq \Lambda_{0}^{-1}\left|v_{0}\right|^{-4 /\left(4-g_{s}\right)}
$$

In the limit $g_{s} \rightarrow 4$ from below the correlation length (27) diverges which is in correspondence with the wellknown fact 17 that the model (14) has a phase transition at $g_{s}=4$. We also note that for the case of noninteracting electrons, $g_{c, s}=2$, the energy scale $\hbar / L_{c}$ corresponding to (27) has been identified earlier ${ }^{1}$; a related renormalization-type argument is given in Section IIIC.

At the scales exceeding $L_{c}$ the spin fluctuations are effectively frozen and no longer contribute into the groundstate energy. Thus the latter can be estimated by substituting $L \simeq L_{c}$ in Eqs.(25) and (26). As a result for $2<g_{s}<4$ the correction to the ground-state energy $\delta E$ is estimated as

$$
\delta E \simeq-\frac{v_{0}^{2} \hbar \Lambda_{0}}{2} B\left(g_{s}\right)\left(1-\frac{\left|v_{0}\right|^{2\left(g_{s}-2\right) /\left(4-g_{s}\right)}}{g_{s}}\right)
$$

while for $g_{s}=2$ we find

$$
\delta E=-\frac{v_{0}^{2} \hbar \Lambda_{0}}{2 \gamma} \ln \left(\frac{1}{v_{0}^{2}}\right)
$$

The numerical uncertainty present in the definition of the characteristic scale $L_{c}$, Eq. (27), translates into an uncertainty of the term of Eq. (28) non-analytic in the parameter $v_{0}$ : here $v_{0}$ should be viewed as $v_{0}$ times a constant of order unity. However the analytic $v_{0}^{2}$ term is given exactly. At the same time Eq.(29) continues to possess logarithmic accuracy as the uncertainty of $L_{c}$ only affects the argument of the logarithm.

Since the parameter $v_{0}$, Eq. (15), vanishes at halfinteger dot population $N$, the ground-state energy given by Eqs. (28) and (29) and related equilibrium properties exhibit non-analytic behavior at half-integer $N$. For example for non-interacting electrons, $g_{c, s}=2$ (or more generally in the presence of $S U(2)$ spin symmetry, $\left.g_{s}=2\right)$, the capacitance of the $\operatorname{dot} C \propto \partial^{2} E / \partial N^{2}$ diverges logarithmically at half-integer dot population, the result derived by Matveev through an exact calculation ${ }^{6}$.

More generally, $2<g_{s}<4$, the singular part of the energy behaves as $\delta E_{\text {sing }} \propto|\delta N|^{4 /\left(4-g_{s}\right)}$ (see Eq.(28)) where $\delta N$ is a small deviation from a half-integer dot population. Although the corresponding contribution into the capacitance $C_{\text {sing }} \propto|\delta N|^{2\left(g_{s}-2\right) /\left(4-g_{s}\right)}$ vanishes, the third-order derivative of the energy, $\partial^{3} E_{\text {sing }} / \partial N^{3} \propto$ $|\delta N|^{\left(3 g_{s}-8\right) /\left(4-g_{s}\right)}$ diverges for $g_{s}<8 / 3$. Generally, the spin correlation exponent $g_{s}$ parameterizes the order of 
the phase transition at half-integer population of the dot (i.e. the order of the first divergent derivative of the ground-state energy): it is of the second-order at $g_{s}=2$ and of the $l$ th-order for $g_{s}<4(l-1) / l$ and $l>2$. As $g_{s}$ increases from 2 to 4 , spin fluctuations increase, the non-analyticity of the ground-state energy manifests itself in the divergence of progressively higher-order derivative and the order of the phase transition increases approaching infinity at $g_{s}=4$. The ultimate origin of these singularities is the discreteness of the electron spin. For $g_{s} \geq 4$ spin fluctuations become unbound and the correction to the ground-state energy becomes regular and proportional to $-(\cos 2 \pi N+1)$ (see Eqs.(22) and (15)) still exhibiting remnants of the discreteness of the electrons.

\section{Beyond perturbation theory: renormalization-group analysis}

The heuristic results (28) and (29) can be placed on a firmer ground through a perturbative, $v_{0} \ll 1$, renormalization-group analysis of the effective action (14). It is well-known that renormalization procedure involves repeated elimination of microscopic degrees of freedom $\frac{10}{}$. This process generates terms which renormalize the parameters of the initial action thus bringing about observable physics, and contributions which are irrelevant in the renormalization-group sense and thus can be dropped. For the model (14) the associated terms are discussed in the literature $\frac{17}{}$. Additionally, elimination of microscopic degrees of freedom can generate extensive quantities which (in the present context) represent contributions into the ground-state energy. As far as we know, these contributions which will be our primary concern, have not been discussed which gives us an excuse to summarize the salient steps of the whole calculation.

In the sequel we adopt the approach of Nozières ${ }^{16}$ who investigated the classical problem of the roughening phase transition. As usual, the field $\theta_{s}$ is presented as a sum of "fast", $\theta_{s}^{(f)}$, and "slow", $\theta_{s}^{(s)}$, modes:

$$
\theta_{s}(\tau)=\theta_{s}^{(f)}(\tau)+\theta_{s}^{(s)}(\tau)
$$

Below we will use the sharp cutoff procedure ${ }^{10}$ appropriate for the model (14), i. e. choose

$$
\theta_{s}^{(f)}(\omega)=\theta_{s}(\omega), \quad \Lambda_{0}(1-\epsilon)<|\omega| \leq \Lambda_{0},
$$

where $\epsilon \ll 1$, and

$$
\theta_{s}^{(s)}(\omega)=\theta_{s}(\omega) . \quad|\omega| \leq \Lambda_{0}(1-\epsilon)
$$

After the fast degrees of freedom are eliminated, the effective action (14) turns into a functional of slow modes, $A_{s}\left[\theta_{s}^{(s)}(\tau)\right]$, which to second order in $v_{0}$ is given by the expansion 16,17 :

$$
A_{s}\left[\theta_{s}^{(s)}(\tau)\right]=\pi \hbar \int_{|\omega| \leq \Lambda_{0}(1-\epsilon)} \frac{d \omega}{2 \pi g_{s}}|\omega|\left|\theta_{s}(\omega)\right|^{2}
$$

$$
\begin{aligned}
& +<A_{\text {int }}\left[\theta_{s}^{(s)}(\tau)+\theta_{s}^{(f)}(\tau)\right]>_{f} \\
& -\frac{1}{2 \hbar}<\left(A_{\text {int }}\left[\theta_{s}^{(s)}(\tau)+\theta_{s}^{(f)}(\tau)\right]\right. \\
& \left.-<A_{\text {int }}\left[\theta_{s}^{(s)}(\tau)+\theta_{s}^{(f)}(\tau)\right]>_{f}\right)^{2}>_{f}
\end{aligned}
$$

Here $<>_{f}$ denotes averaging over fast degrees of freedom with the Gaussian probability distribution determined by the action (18) except that the frequency range, according to Eq.(31), is $\Lambda_{0}(1-\epsilon)<|\omega| \leq \Lambda_{0}$.

To first order we find

$$
\begin{aligned}
A_{1} & =-v_{0}\left(1-\frac{\pi^{2}}{2}<\left(\theta_{s}^{(f)}\right)^{2}>_{f}\right) \Lambda_{0} \hbar \int d \tau \cos \pi \theta_{s}^{(s)} \\
& =-v_{0}\left(1-\frac{g_{s}}{4} \epsilon\right) \Lambda_{0} \hbar \int d \tau \cos \pi \theta_{s}^{(s)} \\
& =-v \Lambda \hbar \int d \tau \cos \pi \theta_{s}^{(s)}
\end{aligned}
$$

where we employed the fact that the mean-square fluctuation of the fast modes, $<\left(\theta_{s}^{(f)}\right)^{2}>_{f}$, is given by the integral (19) except that the integration range is $\Lambda_{0}(1-\epsilon)<|\omega| \leq \Lambda_{0}$, Eq.(31). The last representation in (34) combined with the first term of (33) form an action having the same functional form as the original action $A_{s}$, Eq.(14) except for smaller cutoff,

$$
\Lambda=\Lambda_{0}(1-\epsilon),
$$

and a slightly different amplitude of the cosine term,

$$
v=v_{0} \frac{1-g_{s} \epsilon / 4}{1-\epsilon},
$$

To second order we obtain

$$
\begin{aligned}
A_{2}= & -\frac{v_{0}^{2} \Lambda_{0}^{2} \pi^{2} \hbar}{2} \int d \tau d \tau^{\prime} \sin \pi \theta_{s}^{(s)} \sin \pi \theta_{s}^{(s) \prime} \\
& \times<\theta_{s}^{(f)} \theta_{s}^{(f)}{ }^{\prime}>_{f},
\end{aligned}
$$

where the prime means that the argument of corresponding function is $\tau^{\prime}$. The correlation function < $\theta_{s}^{(f)} \theta_{s}^{(f)}{ }^{\prime}>_{f}$ is given by

$$
\begin{aligned}
<\theta_{s}^{(f)} \theta_{s}^{(f)}{ }^{\prime}>_{f} & =\frac{g_{s}}{2 \pi} \int_{\Lambda_{0}(1-d l)<|\omega| \leq \Lambda_{0}} \frac{d \omega}{2 \pi} \frac{\exp i \omega\left(\tau-\tau^{\prime}\right)}{|\omega|} \\
& =\frac{g_{s}}{2 \pi^{2}} \epsilon \cos \left(\Lambda_{0}\left(\tau-\tau^{\prime}\right)\right),
\end{aligned}
$$

which allows us to rewrite Eq.(37) in a more explicit form:

$$
\begin{aligned}
A_{2} & =\frac{v_{0}^{2} \Lambda_{0}^{2} \hbar g_{s}}{8} \epsilon \int d \tau d \tau^{\prime} \cos \left(\Lambda_{0}\left(\tau-\tau^{\prime}\right)\right) \\
& \times\left(\cos \pi\left(\theta_{s}^{(s)}+\theta_{s}^{(s) \prime}\right)-\cos \pi\left(\theta_{s}^{(s)}-\theta_{s}^{(s)}{ }^{\prime}\right)\right)(39)
\end{aligned}
$$

A further truncation is necessary to see the implications of Eq. (39). First, the $\cos \pi\left(\theta_{s}^{(s)}+\theta_{s}^{(s)}{ }^{\prime}\right)$ term can be dropped since for $\tau=\tau^{\prime}$ it becomes the second harmonic 
of the main $\cos \pi \theta_{s}^{(s)}$ oscillation, and thus is irrelevant in the renormalization-group sense.

Gradient expansion of the other term

$$
\begin{aligned}
\cos \pi\left(\theta_{s}^{(s)}-\theta_{s}^{(s) \prime}\right) & \approx 1-\frac{\pi^{2}}{2}\left(\theta_{s}^{(s)}-\theta_{s}^{(s){ }^{\prime}}\right)^{2} \\
& \approx 1-\frac{\pi^{2}}{2}\left(\left(\tau-\tau^{\prime}\right) d \theta_{s}^{(s)} / d \tau\right)^{2}(40)
\end{aligned}
$$

is not appropriate for large magnitude of the difference $\tau-\tau^{\prime}$ where the two values $\theta_{s}^{(s)}$ and $\theta_{s}^{(s)}$ ' are hardly correlated: the right-hand side of (40) needs a factor specifying the extent to which these values are correlated. The same situation is encountered in the context of the usual sineGordon theory where Knops and den Ouden $\frac{18}{}$ demonstrated that continuing the expansion of the cosine to all orders generates the necessary factor. Below we adopt an equivalent physical argument due to Nozières $\underline{16}$, and go beyond the existing treatments of the action (14):

The field $\theta_{s}^{(s)}$ in (40) should be considered as a sum of the equilibrium fluctuation $\theta_{s}^{(s)}$ and a small correction $\zeta$ (which might be a response to a small low-frequency perturbation). Thus instead of (40), first we average over equilibrium fluctuations which is followed by the expansion in $\zeta$. This generates the expansion replacing Eq. (40):

$$
\begin{aligned}
& \cos \pi\left(\theta_{s}^{(s)}-\theta_{s}^{(s) \prime}\right) \\
\rightarrow & \left(1-\frac{\pi^{2}}{2}\left(\zeta-\zeta^{\prime}\right)^{2}\right)<\cos \pi\left(\theta_{s}^{(s)}-\theta_{s}^{(s) \prime}\right)>_{0 s} \\
\approx & \left(1-\frac{\pi^{2}}{2}\left(\left(\tau-\tau^{\prime}\right) d \theta_{s}^{(s)} / d \tau\right)^{2}\right) \\
\times & \exp \left(-\frac{\pi^{2} \chi\left(\tau-\tau^{\prime}\right)}{2}\right)
\end{aligned}
$$

We note that the same correlation exponential appears in the second-order result, Eq.(22) . > From technical viewpoint, the presence of this factor eliminates all the potential difficulties usually encountered when sharp cutoff procedures are used $\mathrm{d}^{10,12}$.

Insertion of the expansion (41) back into (39) makes it clear that a small positive contribution of the $\int d \tau\left(d \theta_{s}^{(s)} / d \tau\right)^{2}$ form is generated under renormalization. For the classical problem of the roughening phase transition such contributions are important parts of the physics as they renormalize the interface stiffness $\frac{16}{}$. However in the present context they are irrelevant and thus can be dropped 17 . For the model (14) the role of the stiffness is played by $g_{s}^{-1}$ which does not renormalize to any order ${ }^{17}$ : renormalization procedure cannot generate non-analytic contributions proportional to $|\omega|$.

The constant term in (41) generates a small negative correction to the ground-state energy

$$
\Delta E=-\frac{v_{0}^{2} \Lambda_{0} \hbar}{2} H\left(g_{s}\right) \epsilon,
$$

where the function

$$
H\left(g_{s}\right)=\frac{g_{s}}{2} \int_{0}^{\infty} d t \cos t \exp \left(-\frac{g_{s}}{2} \operatorname{Cin}(t)\right)
$$

is defined unambiguously for $g_{s} \geq 2$. Using the definition of the cosine integral (21) this formula can be rewritten as

$$
\begin{aligned}
H\left(g_{s}\right) & =\frac{g_{s}}{2} \int_{0}^{\infty}\left(1-t \frac{d(\operatorname{Cin}(t))}{d t}\right) \exp \left(-\frac{g_{s}}{2} \operatorname{Cin}(t)\right) d t \\
& =\int_{0}^{\infty} t^{1-g_{s} / 2} d\left(t^{g_{s} / 2} \exp \left(-\frac{g_{s}}{2} \operatorname{Cin}(t)\right)\right)
\end{aligned}
$$

which implies

$$
H(2)=\lim _{t \rightarrow \infty}(t \exp (-\operatorname{Cin}(t)))=\gamma^{-1} \approx 0.5615
$$

This demonstrates the special role played by $g_{s}=2$ only in the presence of $S U(2)$ spin symmetry is the corresponding value of $H\left(g_{s}\right)$ controlled by the $t \rightarrow \infty$ limit.

For $g_{s}>2$ we integrate Eq. (44) by parts which establishes a relationship with the function $B\left(g_{s}\right)$, Eq.(23), appearing in the second-order expression (22):

$$
H\left(g_{s}\right)=\left(\frac{g_{s}}{2}-1\right) B\left(g_{s}\right)
$$

Renormalization-group transformation which led to Eqs. (35), (36), and (42) can be carried over repeatedly by reducing the frequency cutoff by successive infinitesimally small amounts $\epsilon=d l \rightarrow 0$. Since each step of the transformation incorporates the effect of previous renormalizations, the outcome is customary to represent in the form of differential flow equations:

$$
\begin{gathered}
\frac{d \Lambda}{d l}=-\Lambda \\
\frac{d v}{d l}=\left(1-\frac{g_{s}}{4}\right) v \\
\frac{d E}{d l}=-\frac{v^{2} \Lambda \hbar}{2} H\left(g_{s}\right)
\end{gathered}
$$

These equations have to be solved subject to the "initial" conditions, $\Lambda(l=0)=\Lambda_{0}, v(l=0)=v_{0}$; the macroscopic behavior is recovered in the $\Lambda(l \rightarrow \infty) \rightarrow 0$ limit.

Eq.(47) whose solution is given by

$$
\Lambda(l)=\Lambda_{0} e^{-l}
$$

is a common place in renormalization-group calculations.

Eq.(48) and its solution

$$
v(l)=v_{0} e^{\left(1-g_{s} / 4\right) l}
$$

are also well-known ${ }^{17}$ from renormalization-group treatments of the model (14):

For $g_{s}>4$ the parameter $v(l)$ flows to zero as $l \rightarrow \infty$ thus implying that the last term of (14) is irrelevant in renormalization-group sense. This is the region of applicability of perturbative approach with unbound spin 
fluctuations on the dot (see Eq.(19)). For $g_{s}<4$ the parameter $v(l)$ grows under renormalization indicating that the discreteness of the electron spin is relevant and that perturbative treatment fails at low frequencies. As a result the fluctuations of the spin of the dot will be suppressed at flow parameters exceeding a characteristic value $l_{c}$ such as $\left|v\left(l_{c}\right)\right| \simeq 1$ :

$$
l_{c} \simeq \frac{4}{4-g_{s}} \ln \left(\frac{1}{\left|v_{0}\right|}\right)
$$

We note that the corresponding scale in imaginary time direction $\Lambda_{c}^{-1}=\Lambda_{0}^{-1} e^{l_{c}}$ coincides with the heuristic result (27) - the argument that led to (27) is equivalent to the first-order renormalization-group calculation.

Eq.(49) describing renormalization of the ground-state energy due to eliminated high-frequency modes is novel; its counterpart in the context of the conventional sineGordon problem is known ${ }^{12,16}$. In order to compute the contribution into the ground-state energy we first substitute the solutions (50) and (51) in Eq.(49) which turns it into a form

$$
\frac{d E}{d l}=-\frac{v_{0}^{2} \Lambda_{0} \hbar}{2} H\left(g_{s}\right) e^{\left(1-g_{s} / 2\right) l}
$$

that makes explicit a special role played by $S U(2)$ spin symmetry, $g_{s}=2$.

For $g_{s} \geq 4$ spin fluctuations of all frequencies contribute into the ground-state energy thus implying that Eq.(53) should be integrated between zero and infinity. Combined with Eq.(46), it then reproduces the perturbative result (22).

For $2 \leq g_{s}<4$ spin fluctuations are suppressed at small frequencies and the contribution into the groundstate energy can be found by integrating Eq. (53) between zero and the characteristic flow parameter $l_{c}$, Eq.(52). As a result for $2<g_{s}<4$ we find

$$
\delta E \simeq-\frac{v_{0}^{2} \Lambda_{0} \hbar}{2} B\left(g_{s}\right)\left(1-\left|v_{0}\right|^{2\left(g_{s}-2\right) /\left(4-g_{s}\right)}\right)
$$

This is very similar to the heuristic result (28), and the uncertainty of the second term of (54) having its origin in the uncertainty built into the definition of the characteristic flow parameter $l_{c}$, Eq.(52), makes Eqs.(28) and (54) practically indistinguishable.

Finally, for $g_{s}=2$ integrating Eq. (53) between zero and $l_{c}$, Eq.(52), and taking into account the numerical value $H(2)$, Eq.(45), reproduces the heuristic result (29).

\section{Analogy to the sine-Gordon theory}

The main conclusion of previous analysis is that in the free-fermionic/SU(2) symmetric limit, $g_{s}=2$, the ground-state energy (as a function of deviation $\delta N$ away from half-integer dot population) exhibits logarithmic non-analyticity, $\delta E_{\text {sing }} \propto \delta N^{2} \ln (1 /|\delta N|)$; in the interacting case, $2<g_{s}<4$, this is replaced by a power law, $\delta E_{\text {sing }} \propto|\delta N|^{4 /\left(4-g_{s}\right)}$. This outcome is not unique to the zero-dimensional field theory (14) describing the dynamics of nearly-open quantum dot - the conventional one-dimensional sin-Gordon field theory shows the same effect. The pertinent physical phenomenon here is the Peierls instability 19 :

More than fifty years ago Peierls demonstrated that a one-dimensional metal is subject to a spontaneous lattice distortion that converts it into an insulator. The distortion causes commensuration of electronic and ionic subsystems which leads to the reduction of the electronic part of the system energy. For non-interacting electrons Peierls found that the energy gain of commensuration as a function of small lattice distortion $\delta$ has non-analytic form $\delta E_{\text {sing }} \propto \delta^{2} \ln (1 / \delta)$. Since other effects favoring regular lattice spacing $\delta=0$ increase the energy by an amount proportional to $\delta^{2}$, the distortion is inevitable.

Since the Peierls phenomenon just barely occurs for non-interacting electrons, interelectron interactions have a drastic effect on the fate of the instability. For spinless electrons the phenomenological theory of the effect which takes into account interactions, has been given in the past ${ }^{20}$. There it was demonstrated that the electronic energy gain due to the lattice distortion is given by the ground-state energy corresponding to the sineGordon action

$$
A=\int d x d \tau\left(\frac{\rho}{2}\left(\left(\frac{\partial u}{\partial \tau}\right)^{2}+c^{2}\left(\frac{\partial u}{\partial x}\right)^{2}\right)-\lambda \cos 2 \pi n u\right)
$$

Here $u(x, \tau)$ is the electron displacement field, $\rho$ is the mass density, $c$ is the sound velocity, $n$ is the number density, and $\lambda$ is proportional to the amplitude of the lattice distortion $\delta$. The effect of interelectron interactions can be parameterized by dimensionless combination

$$
g=\frac{\pi \hbar n^{2}}{\rho c}
$$

which can be recognized as the "spinless" counterpart of the charge correlation exponent $g_{c}(3)$. Renormalizationgroup analysis of the action (55) resembling that of previous subsection then leads to the following conclusions 20 :

For non-interacting electrons, $g=1$, the form, originally derived by Peierls $\frac{19}{}, \delta E_{\text {sing }} \propto \delta^{2} \ln (1 / \delta)$, is found. This is analogous to the "non-interacting" result, Eq.(29).

In the presence of interactions satisfying $g<2$ (and excluding $g=1$ ), the singular part of the ground-state energy as a function of the lattice distortion $\delta$ is given by $\delta E_{\text {sing }} \propto \delta^{2 /(2-g)}$. This is analogous to our result $\delta E_{\text {sing }} \propto|\delta N|^{4 /\left(4-g_{s}\right)}$ (see Eqs. (28) and (54)).

One of the important differences between the actions (14) and (55) is that the spin correlation exponent $g_{s}$ (3) controlling the degree of zero-point motion in (14) does not renormalize. For the sin-Gordon action (55) the same role is played by the exponent $g$ (56) which does renormalize. However for $g<2$ and small distortion $($ small $\lambda)$ this renormalization is negligible ${ }^{20}$. That is why an analogy can be drawn between the ground-state properties of the field theories (14) and (55). 


\section{NEARLY-CLOSED DOT}

Although the analysis of Section III makes it clear that the logarithmic divergence of the capacitance at halfinteger dot population ${ }^{6}$ is a consequence of assumption that the electrons in the constriction are non-interacting, it cannot explain why the same type of singularity may be present in the weak-tunneling regime $e^{\underline{4}}$ and possibly for any tunneling ${ }^{6}$. To address these issues, we turn our attention to the limit of a nearly-closed quantum dot.

The starting point of the analysis is clarification of the nature of the classical ground state described by the effective action (7). This is determined by the minima of the potential $V\left(\theta_{c}, \theta_{s}\right)$, Eq. (8), and has been discussed by Kane and Fisher $\underline{11}$ in a related context of resonant tunneling of spinless electrons confined between two barriers in the presence of a gate. Although the potential entering Eq.(10) is not quantitatively correct for a nearly-closed dot, it nevertheless retains the symmetries of the problem and can be used as an illustration of the argument below disregarding the $|r| \ll 1$ constraint. Visual aid is provided by Fig. 1 which parallels Figure 2 of Kane and Fisher 11 .

Without the charging term, $E_{c}=0$, the classical ground-state is degenerate with infinitely sharp minima located at integer values of the dot population $\theta_{c}$, which reflects the discreteness of the electrons. The charging term of (8) lifts this degeneracy so that for most values of the dimensionless gate voltage $N$ there is a unique ground state determined by an integer $\theta_{c}$ closest to $N$ with energy gaps of the order $E_{C}$ or larger to the states of higher or lower dot population.

For half-integer values of the dimensionless gate voltage $N$ the ground state is doubly degenerate. Since all other integer population states are separated from these two by energy gaps, it seems plausible ${ }^{4,11}$ that they can be eliminated from the discussion of the hopping dynamics when the dot is slightly open and charge and spin fluctuations are included. The validity of this two-state approximation will be discussed below but for now we assume that it is justified. Then the $\theta_{c}$ coordinates of the minima of the effective potential $V\left(\theta_{c}, \theta_{s}\right)$ are either $N+1 / 2$ or $N-1 / 2$ while their $\theta_{s}$ positions are integers with the constraint that for every minimum its $\theta_{c}$ and $\theta_{s}$ coordinates have the same parity. This means that if nearest minima are connected by straight line segments (see Fig. 1), they will form an infinite zigzag running on average parallel to the $\theta_{s}$ axis.

Opening the dot means that the barriers separating degenerate states of integer population are no longer infinitely high - the effective potential will now have degenerate minima whose $\theta_{c}$ positions are either $N+q / 2$ or $N-q / 2$ where $q$ is the distance between the minima of $V$ along the $\theta_{c}$ axis ${ }^{21}$. At the same time the step of the zigzag in the $\theta_{s}$ direction which is the symmetry of the problem remains unchanged. Including charge and spin fluctuations, i. e. allowing finite $g_{c, s}$, Eq. (3), has the same effect of downward renormalization of the distance

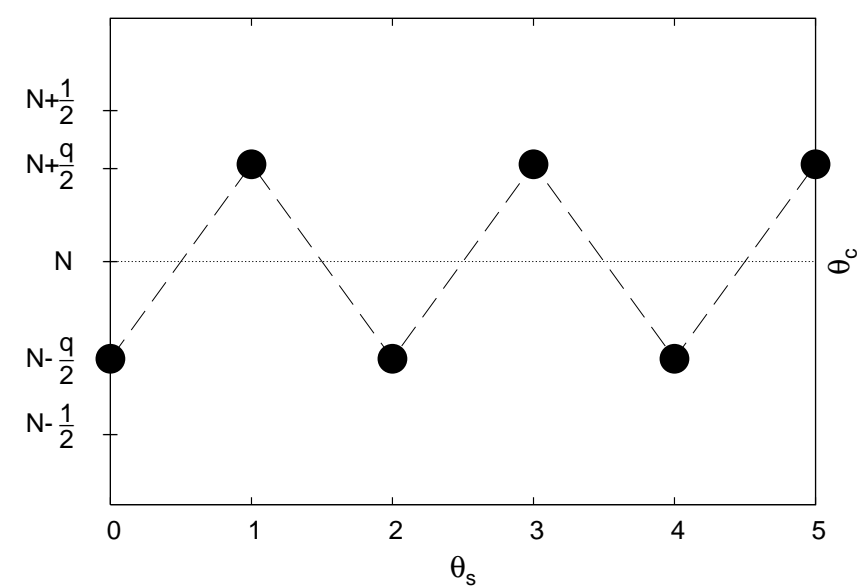

Figure 1: Positions of the degenerate minima of the potential $V\left(\theta_{c}, \theta_{s}\right)$ entering Eq. (7) in the $\left(\theta_{c}, \theta_{s}\right)$ plane for half-integer dimensionless gate voltage $N$. For the purpose of illustration, nearest minima are connected by dashed line segments. The parameter $q$ has a meaning of the distance between the minima in the $\theta_{c}$ direction. Although $q=1$ for a nearly-closed dot, zero-point motion renormalizes it to a $0 \leq q<1$ value. In the drawing we assumed (without the loss of generality) that $N+1 / 2$ is odd.

between the minima of $V$ along the $\theta_{c}$ direction. Indeed, even for a nearly-closed quantum dot where $q=1$ initially, quantum-mechanical tunneling between nearest degenerate ground-states reduces it to a $0 \leq q<1$ value. For $q=0$ the effective potential energy evaluated at $\theta_{c}=N$ is $\theta_{s}$-independent. We note that observable value of the parameter $q$ corresponds to the jump of the dot population as the dimensionless gate voltage $N$ is swept across a half-integer value. Then $q=0$ means that the Coulomb staircase is smeared.

The elementary act of tunneling between nearest ground states consists of a change of the $\theta_{c}$ coordinate by $q$ accompanied by simultaneous change of $\theta_{s}$ by unity. The zigzag sequence of the ground states implies that the tunneling event $\Delta \theta_{c}= \pm q$ must be followed by another tunneling event consisting in opposite sign change of $\Delta \theta_{c}=\mp q$. At the same time tunneling changes of $\theta_{s}$ by unity are not correlated.

To gain better understanding of the physics in the regime of a nearly-closed quantum dot we invoke a mapping ${ }^{12}$ between zero-dimensional quantum field theory defined by the action (7) and that of one-dimensional classical statistical mechanics. Similar to the observation made for the spinless version of the problem ${ }^{7}$, Eq. (7) can be viewed as an effective Hamiltonian of one-dimensional classical statistical mechanics problem involving fluctuating two-component field $\theta_{c, s}$ with imaginary time $\tau$ playing a role of fictitious space direction. The $|\omega|$ dependence of the first term of Eq. (7) implies a $1 / \tau^{2}$ interaction between the fields $\theta_{c, s}$ separated by distance $\tau$.

If not for the presence of the spin field $\theta_{s}$, then for half-integer $N$ the classical statistical mechanics problem 
in question would be identical to that of a ferromagnetic Ising model with $1 / \tau^{2}$ interactions in zero magnetic field. Similar to the Ising model there could be two phases:

In the ordered (broken symmetry) phase the system, initially prepared in one of the minima of the potential $V\left(\theta_{c}, \theta_{s}\right)$, will reside in there indefinitely long despite occasional excursions induced by thermal fluctuations into neighboring minima followed by returns into the original minimum. The excursion is a nucleus of neighboring ground state constricted by two domain walls having opposite signs of the change $\Delta \theta_{c}$. For the Ising model with $1 / \tau^{2}$ interactions the domain walls are known to be responsible for the destruction of $\operatorname{order}^{22}$; the same is expected to hold for the model (7). Domain walls have their quantum-mechanical counterparts in instantons extremal solutions of the equations of classical dynamics in imaginary time satisfying the condition that as imaginary time $\tau$ traverses from minus infinity to infinity, the instanton interpolates between degenerate ground states.

The similarity to the Ising model also implies that the broken symmetry phase of (7) may be removed by proliferating domain walls. As a result the system will freely hop through the minima of $V\left(\theta_{c}, \theta_{s}\right)$ leading to the destruction of the Coulomb staircase in the quantummechanical context.

In order to understand quantitatively the role of the domain walls we find it useful to rewrite the action (7) in an equivalent form which abandons the Fourier representation of the first term and shows explicitly that the latter vanishes for the uniform system:

$$
\begin{aligned}
A_{e f f} & =-\hbar \sum_{\nu=c, s} g_{\nu}^{-1} \int \dot{\theta}_{\nu}(\tau) d \tau \ln \left(\left|\tau-\tau^{\prime}\right| \Lambda_{0}\right) \dot{\theta}_{\nu}\left(\tau^{\prime}\right) d \tau^{\prime} \\
& +\int d \tau V\left(\theta_{c}, \theta_{s}\right)
\end{aligned}
$$

where the integration in the first term is limited by the condition $\left|\tau-\tau^{\prime}\right| \Lambda_{0} \geq 1$ and the dots indicate derivatives.

The domain wall of the field theory (57) can be found by solving equations $\delta A_{e f f} / \delta \theta_{c, s}=0$ subject to the boundary conditions $\theta_{c}(-\infty)-\theta_{c}(\infty)= \pm q$ and $\theta_{s}(-\infty)-\theta_{s}(\infty)= \pm 1$. In the limit of large barriers the solution will have a form of a steep rounded step which can be approximated by

$$
\dot{\theta}_{c}(\tau)= \pm q \delta\left(\tau-\tau_{0}\right), \quad \theta_{s}(\tau)= \pm \delta\left(\tau-\tau_{0}\right)
$$

where $\tau_{0}$ is arbitrary position of the domain wall. The combination of Eq.(57) and (58) implies that in the largebarrier limit the physics is dominated by thermally excited logarithmically interacting domain walls whose sequence is constrained by the zigzag locations of classical ground states discussed earlier.

\section{A. The "entropy" argument of Landau, Thouless and Kosterlitz and Thouless}

We begin with a semi-quantitative argument similar to the "entropy" arguments of Landau ${ }^{23}$, Thouless 22 , and
Kosterlitz and Thouless ${ }^{24}$. The energy of a single domain wall diverges with the system size $L$, as indicated by Eqs. (57) and (58): $E=\hbar\left(q^{2} g_{c}^{-1}+g_{s}^{-1}\right) \ln \left(\Lambda_{0} L\right)$. The entropy is given by the logarithm of the number of ways to put the domain wall on the line: $S=\ln \left(\Lambda_{0} L\right)$. Therefore we find for the change of the free energy $F=E-T S$ for the introduction of a single domain wall

$$
F=\left(\hbar\left(\frac{q^{2}}{g_{c}}+\frac{1}{g_{s}}\right)-T\right) \ln \left(\Lambda_{0} L\right)
$$

The free energy changes sign at the temperature

$$
T_{c}=\hbar\left(\frac{q^{2}}{g_{c}}+\frac{1}{g_{s}}\right)
$$

This suggests that above $T_{c}$ the ground-state is unstable against spontaneous creation of domain walls. Below $T_{c}$ there are only bound pairs of domain walls while above $T_{c}$ there are free domain walls.

To see the implications of these conclusions for the problem of a nearly-closed Coulomb-blockaded quantum dot we note that in the mapping between quantum field-theoretical models and those of classical statistical mechanics ${ }^{12}$ zero-point motion corresponds to thermal fluctuations, i. e. the role of Planck's constant $\hbar$ is played by the temperature $T$. Therefore provided

$$
1-\frac{1}{g_{s}}-\frac{q^{2}}{g_{c}}<0
$$

weak tunneling will not smear the Coulomb staircase. If this is the case, then there is non-zero lower limit to the magnitude of the population jump $\Delta \theta_{c}$ (given by $q$ ) at half-integer dimensionless gate voltage $N$ :

$$
\left(\Delta \theta_{c}\right)_{\min }=\left(g_{c}\left(1-g_{s}^{-1}\right)\right)^{1 / 2}
$$

This parallels the conclusion that there is non-zero lower limit to the magnetization of the one-dimensional classical Ising model with $1 / \tau^{2}$ interactions 22 . A conclusion qualitatively similar to (62) has been found earlier for the spinless version of the Coulomb blockade problem 7 . One may argue that a formal transition from spinfull to spinless electrons would consist in imposing $S U(2)$ spin symmetry, $g_{s}=2$, followed by the replacement ${ }^{9} g_{c}=2 g$. Then Eq. (62) will exactly reproduce the spinless result ${ }^{7}$.

In the presence of $S U(2)$ spin symmetry, $g_{s}=2$, the criterion (61) becomes $g_{c}<2 q^{2}$ which for nearly closed dot, $q=1$, further simplifies to $g_{c}<2$. The latter condition is satisfied in the presence of short-range repulsion between the electrons ${ }^{9}$. We conclude that repulsive interactions between the electrons aid the Coulomb staircase to survive disordering effect of the zero-point motion. This meets the physical expectation as repulsions decrease quantum fluctuations in the charge channel, thus making the system more classical.

It is curious that non-interacting electrons, $g_{c, s}=2$, and a nearly-closed dot, $q=1$, correspond to the sign of equality in Eq. (61) - the "entropy" argument predicts 
neutral stability. This is the first indication that the freeelectron case has to be treated with special care. Here the analysis has to be conducted along two directions:

First, renormalization-group analysis of Section IVB improves on the "entropy" argument, thus allowing us to make definite conclusions about the case of noninteracting electrons. Second, one should try to go beyond the two-state approximation which is in the heart of the zigzag nature of the classical ground states. This more complicated problem will be left for future study.

In the case of inequality opposite to (61), even weak tunneling destroys the Coulomb blockade.

\section{B. Renormalization-group treatment}

One can improve on the "entropy" argument via a renormalization-group analysis of the action (57) perturbative in dimensionless tunneling amplitude $\Delta$ (measured in units of $\hbar \Lambda_{0}$ ) between nearest ground states. Another pertinent parameter is the distance $q$ between the nearest wells of the potential $V\left(\theta_{c}, \theta_{s}\right)$ in the $\theta_{c}$ direction. Corresponding lowest order renormalization-group flow equations are implicit in the work of Kane and Fisher 11 :

$$
\begin{gathered}
\frac{d \Delta}{d l}=\left(1-\frac{1}{g_{s}}-\frac{q^{2}}{g_{c}}\right) \Delta \\
\frac{d q^{2}}{d l}=-8 q^{2} \Delta^{2}
\end{gathered}
$$

If we set ${ }^{25} g_{c}=g_{s}=4 g$, then Eqs.(63) and (64) reduce to those of Kane and Fisher ${ }^{11}$ describing the physics of resonant tunneling of interacting spinless electrons onto and off a dot formed by two barriers in the presence of a gate which couples to the electrons between the barriers.

On the other hand, setting $g_{s}=2$ and $g_{c}=2 g$ nearly reproduces renormalization-group equations describing spinless version of the problem of nearly-closed Coulombblockaded quantum $\operatorname{dot}^{7}$; the physics encoded in Eqs.(63) and (64) parallels in many ways that of the spinless case.

Comparing Eqs.(61) and (63) we conclude that the "entropy" argument of Section IVA is equivalent to firstorder renormalization-group calculation establishing the range of stability of the zero-tunneling fixed line.

Eqs. (63) and (64) have to be solved subject to the "initial" conditions, $\Delta(l=0)=\Delta_{0}$ and $q(l=0)=q_{0}$; the values of $\Delta_{0}$ and $q_{0}$ can be computed from the form of the action (57).

The flow diagram corresponding to Eqs.(63) and (64) is sketched in Fig.2. As typical of the Kosterlitz-Thouless phase transition 24 , the flows can be divided into two main regions - presently they correspond to the loci of the models where the Coulomb blockade either survives disordering effect of zero-point motion or ceases to exist.

In the former case the systems satisfying $1-g_{s}^{-1}-$ $q_{0}^{2} / g_{c} \leq 0$ (compare with Eq.(61) ) and $4 \Delta_{0}^{2} \leq q_{0}^{2} / g_{c}+$ $g_{s}^{-1}-1-\left(1-g_{s}^{-1}\right) \ln \left(q_{0}^{2} / g_{c}\left(1-g_{s}^{-1}\right)\right)$, i. e. those to

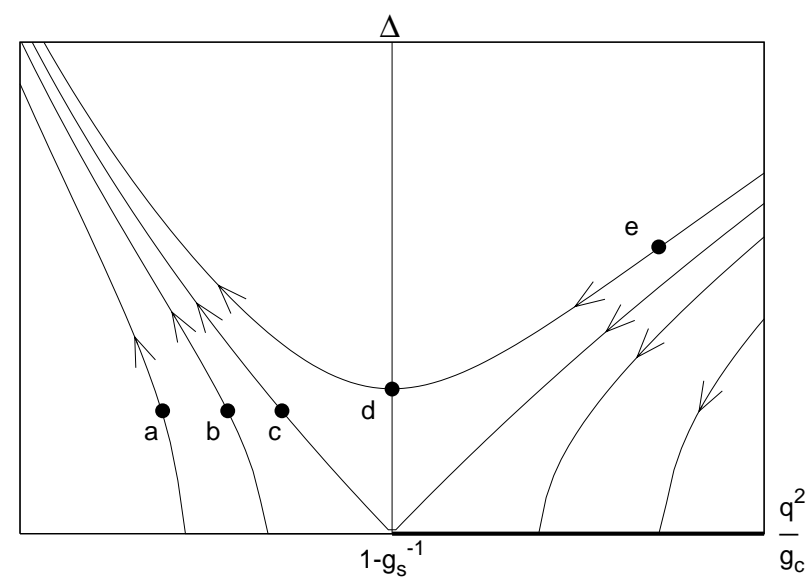

Figure 2: The flow diagram corresponding to Eqs. (63) and (64). The stable part of the $\Delta=0$ fixed line is shown in bold, and the arrows indicate the direction of the flow. The models whose loci are given by the "initial" conditions "a"-"e" all exhibit smeared Coulomb staircase with characteristic flow parameter $l_{c}$, given by Eqs. (65)-(69), respectively. Specifically, non-interacting electrons, $g_{c . s}=2$, entering a nearly-closed dot, $q_{0}=1$, are represented by the "initial" condition 'd".

the right of the separatrix $4 \Delta^{2}=q^{2} / g_{c}+g_{s}^{-1}-1-$ $\left(1-g_{s}^{-1}\right) \ln \left(q^{2} / g_{c}\left(1-g_{s}^{-1}\right)\right)$ are carried by the flow to the stable fixed line, $\Delta=0,1-g_{s}{ }^{-1}-q^{2} / g_{c} \leq 0$. This indicates that tunneling is irrelevant in macroscopic limit, and the discontinuity of the dot population, $\Delta \theta_{c}$ (given by $q$ ), at half-integer $N$ satisfies the inequality $\left(\Delta \theta_{c}\right)_{\min } \geq\left(g_{c}\left(1-g_{s}^{-1}\right)\right)^{1 / 2}$. The equality is reached at the Kosterlitz-Thouless phase transition point, $4 \Delta_{0 c}^{2}=q_{0}^{2} / g_{c}+g_{s}^{-1}-1-\left(1-g_{s}^{-1}\right) \ln \left(q_{0}^{2} / g_{c}\left(1-g_{s}^{-1}\right)\right)$, where the population jump (62) depends only on interparticle interactions. This universal relationship which is a general feature of a Kosterlitz-Thouless transition ${ }^{24}$ is analogous to that between the magnetization jump, the amplitude of the $1 / \tau^{2}$ interaction and the phase transition temperature of the Ising model ${ }^{22}, 26$.

The systems to the left of the separatrix $4 \Delta^{2}=q^{2} / g_{c}+$ $g_{s}^{-1}-1-\left(1-g_{s}^{-1}\right) \ln \left(q^{2} / g_{c}\left(1-g_{s}^{-1}\right)\right)$ are taken by the flow outside of the range of applicability of Eqs.(63) and (64), the effective tunneling amplitude, $\Delta$, is seen to increase while $q$, the distance between the minima of the potential $V\left(\theta_{c}, \theta_{s}\right)$ in the $\theta_{c}$ direction, tends to zero. We are thus in the regime of smeared Coulomb staircase with continuous dependence of the dot population on the gate voltage. Although the Kosterlitz-Thouless transition is continuous, in going from sharp to smeared Coulomb staircase, the shape of the staircase changes discontinuously.

In the classical limit of closed dot, $\Delta_{0}=0$, the distance between the minima of the potential $V\left(\theta_{c}, \theta_{s}\right)$ in the $\theta_{c}$ direction is unity, $q_{0}=1$. Then $S U(2)$ symmetric models with electron-electron repulsion, i. e. those satisfying $g_{s}=2, g_{c}<2$, belong to the stable part of the fixed line, $\Delta=0,1-g_{s}{ }^{-1}-q^{2} / g_{c} \leq 0$. Allowing weak tunneling, i. e. sufficiently small $\Delta_{0}$, only renormalizes 
the population jump at half-integer gate voltage $N$ downward so that $0<\Delta \theta_{c}<1$. This supports the conclusion of Section IVA that weak tunneling does not destroy the Coulomb blockade in the presence of interparticle repulsions. However at sufficiently large tunneling amplitude $\Delta_{0 c}$ the Kosterlitz-Thouless transition takes place, and for $\Delta_{0}>\Delta_{0 c}$ the Coulomb staircase ceases to exist.

In this same classical limit, $\Delta_{0}=0, q_{0}=1$, but with the electrons in the channel satisfying $1-g_{s}{ }^{-1}-g_{c}^{-1}>$ 0 , we find ourselves on the unstable part of the $\Delta=0$ fixed line. Here allowing infinitesimally small tunneling, $\Delta_{0}$, immediately destroys the classical staircase which is consistent with the "entropy" argument.

The free-electron case, $g_{c, s}=2$, is marginal because classically, $\Delta_{0}=0, q_{0}=1$, it corresponds to the end point of the zero-tunneling, $\Delta=0$, stable fixed line. However allowing infinitesimally small tunneling, as indicated in Fig.2, immediately moves it into the regime where the tunneling amplitude grows under renormalization, and thus the Coulomb blockade is destroyed. This is in agreement with the original result ${ }^{4}$.

The systems where the Coulomb blockade is destroyed, i. e. those which under renormalization transformation are taken outside the range of applicability of perturbative, $\Delta \ll 1$, equations (63) and (64), can be characterized by the characteristic flow parameter $l_{c}$ for which $\Delta\left(l_{c}\right) \simeq 1$. The corresponding frequency scale, $\Lambda_{c}=$ $\Lambda_{0} e^{-l_{c}}$ (see Eq.50) marks the onset of strong charge (electron number) fluctuations on large time scales:

At times shorter than $\Lambda_{c}^{-1}$, the tunneling remains weak, $\Delta(l) \ll 1$, and the dot population is frozen at an integer value. On the contrary, at times larger than $\Lambda_{c}^{-1}$, the tunneling is strong and the population of the dot fluctuates freely (around a half-integer value) thus making the particle discreteness irrelevant. The "spin" counterpart of the parameter $l_{c}$ (see Eq.(52)) plays a related role in marking the onset of freezeout of spin fluctuations pertinent to understanding the physics of nearly-open dot.

The estimate of $l_{c}$ cannot be expected to be more than qualitative as the perturbative equations (63) and (64) are at most only indicative of its presence:

If classically the model in question belongs to the unstable part of the zero tunneling fixed line, then the flow starts out vertically away from the $\Delta=0$ axis. If additionally the beginning point is not too close to the end point $\Delta=0,1-g_{s}^{-1}-q^{2} g_{c}^{-1}=0$ (initial condition "a" in Fig.2), then to lowest order in $\Delta_{0}$, the renormalization of $q$ given by Eq.(64) can be neglected. With this in mind the characteristic flow parameter $l_{c}$ can be found to be

$$
l_{c}=\frac{1}{1-g_{s}^{-1}-g_{c}^{-1}} \ln \left(\frac{1}{\Delta_{0}}\right)
$$

If, on the other hand, the beginning point of the flow is close to the end point $\Delta=0,1-g_{s}^{-1}-q^{2} g_{c}^{-1}=0$ (initial condition "b" in Fig.2), then renormalization of $q$, given by Eq.(64) is no longer negligible; instead we find

$$
l_{c}=\frac{1}{1-g_{s}^{-1}-g_{c}^{-1}} \ln \left(\frac{\left(1-g_{s}^{-1}-g_{c}^{-1}\right)}{\Delta_{0}\left(1-g_{s}^{-1}\right)^{1 / 2}}\right)
$$

We note that the estimates (65) and (66) are only logarithmically sensitive to the uncertainty, $\Delta\left(l_{c}\right) \simeq 1$, built into the definition of the characteristic flow parameter $l_{c}$.

If the beginning point of the flow belongs to the outgoing leftward separatrix in Fig.2 (initial condition "c"), then to lowest order in $\Delta_{0}$ the characteristic flow parameter $l_{c}$ is given by

$$
l_{c}=\frac{1}{2 \Delta_{0}\left(2\left(1-g_{s}^{-1}\right)\right)^{1 / 2}}
$$

while if the initial point is at the minimum of the flow trajectory, $1-g_{s}^{-1}-q^{2} g_{c}^{-1}=0$ (initial condition "d" in Fig.2), then we find

$$
l_{c}=\frac{\pi}{4 \Delta_{0}\left(2\left(1-g_{s}^{-1}\right)\right)^{1 / 2}}
$$

The models located between the initial conditions " $c$ " and "d" in Fig. 2 have characteristic flow parameter $l_{c}$ behaving as $\Delta_{0}^{-1}\left(1-g_{s}^{-1}\right)^{-1 / 2}$ and interpolating between the estimates (67) and (68). We note that the systems represented by the initial condition " $d$ " in Fig. 2 include non-interacting electrons, $g_{c, s}=2$, for which the characteristic flow parameter is $l_{c}=\pi / 4 \Delta_{0}$.

If the model in question lies close to the point of Kosterlitz-Thouless phase transition separating the regimes of sharp and smeared Coulomb staircase, i. e. slightly higher than the incoming rightmost separatrix (initial condition " $\mathrm{e}$ " in Fig. 2), then the effective tunneling rate $\Delta(l)$ initially decreases with $l$ followed by an increase at larger $l$. As a result the characteristic flow parameter

$$
l_{c}=\frac{\pi}{4 \sqrt{\left.\left(1-g_{s}^{-1}\right) \Delta_{0 c}\left(\Delta_{0}-\Delta_{0 c}\right)\right)}}
$$

is dominated by the "time" spent in the vicinity of the end point $\Delta=0,1-g_{s}^{-1}-q^{2} g_{c}^{-1}=0$. Here $\Delta_{0 c}$ is the tunneling amplitude at the phase transition and it is assumed that $\Delta_{0}-\Delta_{0 c} \ll \Delta_{0 c}$. As expected, the corresponding scale in imaginary time direction $L_{c}=\Lambda_{0}^{-1} e^{l_{c}}$ has a functional form characteristic of the behavior of the correlation length in the vicinity of the KosterlitzThouless phase transition 24 .

The curious feature of the leading order estimates (67)- 69) is that they are insensitive to the uncertainty $\Delta\left(l_{c}\right) \simeq 1$ built into the definition of the characteristic flow parameter $l_{c}$.

\section{Vicinity of half-integer dot population: heuristic argument}

One of the most intriguing statements made by Matveev is the argument that (for non-interacting electrons) the weak-tunneling results are implied by those found for a nearly-open $\operatorname{dot} 6$.

Renormalization-group analysis of Section IVB enables us to see how and why the types of non-analytic behavior 
of the ground-state properties found for a nearly-open dot (Section III) may be applicable well beyond that regime:

This is expected to be the case for every system which under renormalization transformation flows outside the range of applicability of perturbative equations (63) and (64), i. e. into the region of large tunneling amplitude $\Delta$ and vanishing distance $q$ between the minima of the potential $V\left(\theta_{c}, \theta_{s}\right)$, Eq.(77). Since this is the regime of a nearly-open dot, it seems plausible that no new physics intervenes for intermediate tunneling. As far the the weak-tunneling regime goes, the description of Section III must take over at frequencies smaller than $\Lambda_{c}=\Lambda_{0} e^{-l_{c}}$.

As a test of this statement we now show how the weak-tunneling results can be guessed from their strongtunneling counterparts. First we remind the reader that as charge fluctuations renormalize the distance $q$ between the zigzag minima of the effective potential $V\left(\theta_{c}, \theta_{s}\right)$ in the $\theta_{c}$ direction, the step of the zigzag in the $\theta_{s}$ direction remains unaffected. The latter periodicity becomes "invisible" for $q=0$ and half-integer dimensionless gate voltage because the effective potential ceases to be dependent on the spin field $\theta_{s}$.

The periodicity with respect to the spin field $\theta_{s}$ becomes "visible" again if the dimensionless gate voltage $N$ is tuned away from half-integer value. For small deviation the effective potential will have be proportional to $\delta N$, and the overall energy scale of the potential will be given by the charging energy $E_{C}$. These arguments imply the estimate

$$
V\left(\theta_{s}\right) \simeq E_{C} \delta N \cos \pi \theta_{s}
$$

where in displaying the $\theta_{s}$ periodicity we restricted ourselves to the leading Fourier harmonic and set zero of $V$ at $\delta N=0$. The potential of exactly the same functional form enters the effective action describing the strongtunneling dynamics of the spin degrees of freedom (14), and the vicinity of a half-integer dimensionless gate voltage, $\delta N \ll 1$, guarantees the applicability of the results of Section III. Keeping in mind that the role of the frequency cutoff $\Lambda_{0}$ will be played by $\Lambda_{c}=\Lambda_{0} e^{-l_{c}}$, we find the correspondence

$$
v_{0} \rightarrow-\frac{E_{C} \delta N}{\hbar \Lambda_{c}} \simeq-\delta N e^{l_{c}}
$$

where we assumed a fixed ratio of the charging energy $E_{C}$ to the original cutoff energy $\hbar \Lambda_{0}$. Therefore the ground-state properties of nearly-closed quantum dot in the vicinity of half-integer dimensionless gate voltage can be extracted from the strong-tunneling results (Section III) provided the substitution (71) is made and the frequency cutoff $\Lambda_{0}$ is replaced with $\Lambda_{c}=\Lambda_{0} e^{-l_{c}}$.

For example, making these changes in Eq.(29) we find that in the presence of $S U(2)$ spin symmetry, $g_{s}=2$, the non-analytic correction to the ground-state energy in the weak-tunneling regime has the form

$$
\delta E \simeq-E_{C} e^{l_{c}} \delta N^{2} \ln \left(\frac{1}{\delta N^{2}}\right)
$$

For non-interacting electrons, when $l_{c}=\pi / 4 \Delta_{0}$ (see Eq.(68)), and to leading order in the tunneling amplitude, this reproduces the functional form derived earlier 4 via the analogy to the two-channel Kondo problem and the exact solution of the latter.

Our analysis implies that non-analytic behavior exhibited in Eq.(72) is also characteristic of any system with $S U(2)$ spin symmetry having not very strong interparticle repulsions and sufficiently large tunneling amplitude. Specifically, in the vicinity of the critical tunneling amplitude $\Delta_{0 c}$ which corresponds to the Kosterlitz-Thouless transition into the regime of sharp Coulomb staircase, the correction to the ground-state energy is given by the combination of Eqs.(69) and (72).

\section{CONCLUSIONS}

In this paper we analyzed the zero-temperature dynamics of electrons hopping onto and off a large quantum dot connected to a reservoir via a one-dimensional channel and interparticle interactions inside the channel as well as Coulomb interactions among the electrons on the dot were taken into account. The main analytical tool applied to understand both the limits of strong and weak tunneling was the renormalization-group method.

For strong tunneling (nearly-open dot) the charge discreteness is irrelevant and the physics is dominated by fluctuating spin degrees of freedom which are responsible for non-analytic behavior of the ground-state properties at half-integer dot population. Specifically, the previously found logarithmic divergence of the dot capacitance ${ }^{6}$ is demonstrated to be a consequence of the assumption that electrons in the are non-interacting. We show that the same conclusion holds for physical systems possessing $S U(2)$ spin symmetry. More generally, however, the logarithmic singularity is replaced by a power law non-analyticity whose exponent is determined by the degree of spin fluctuations.

For strong tunneling (nearly-closed dot) and halfinteger dot population the spin degrees of freedom play a secondary role and the physics is dominated by zeropoint hops of the electrons onto and off the dot. If these fluctuations are sufficiently strong, the Coulomb blockade is destroyed by arbitrarily weak tunneling. Noninteracting electrons belong to this regime which is in agreement with previous studies ${ }^{4}$. If the charge fluctuations are sufficiently weak, then the Coulomb blockade survives provided the tunneling is not too strong. The regimes of sharp and smeared Coulomb staircases meet at a phase transition of the Kosterlitz-Thouless kind.

Renormalization-group analysis of the weak-tunneling regime also makes a case in favor of the argument (first given for non-interacting electrons ${ }^{6}$ ) that non-analytic behavior of the ground-state properties at half-integer dot population found in the limit of nearly-open dot can extend well beyond the regime of strong tunneling.

Finally, renormalization-group treatment of weak tun- 
neling rests on the two-state approximation in which all the population states of the quantum dot separated from the two degenerate minima by energy gaps are ignored. Although physically plausible, similar to the spinless version of the problem ${ }^{7}$, its applicability to the case of noninteracting electrons requires further justification. Indeed, in the classical limit of closed dot, $\Delta_{0}=0, q_{0}=1$, the locus of non-interacting electrons, $g_{c, s}=2$, coincides with the end point of the zero-tunneling fixed line in Fig.2. Allowing small finite tunneling $\Delta_{0}$ brings this model into the unstable regime characterized by the initial condition " $d$ ". If we now assume that the ignored metastable states are populated with small finite probability, then the actual electron number fluctuations will be suppressed stronger than predicted by the two-state approximation.

Heuristically, the effect of weaker zero-point motion can still be seen within the two-state analysis - the initial point of the flow will have to be located somewhat to the right of the initial condition "d" in Fig.2. If the initial condition lies to the right of the incoming separatrix of the flow, then the Coulomb blockade can survive weak tunneling. Additionally, the Coulomb staircase would be destroyed at finite tunneling via a phase transition. This possibility calls for an investigation which avoids the twostate truncation altogether. Variational analysis of this problem which does not rely on the two-state approximation and similar to the one used in the spinless case ${ }^{7}$ will be presented elsewhere ${ }^{27}$.

\section{ACKNOWLEDGMENTS}

This work was supported by the Chemical Sciences, Geosciences and Biosciences Division, Office of Basic Energy Sciences, Office of Science, U. S. Department of Energy, and by the Thomas F. Jeffress and Kate Miller Jeffress Memorial Trust.
1 I. L. Aleiner, P. W. Brouwer, and L. I. Glazman, Phys. Rep. 358, 309 (2002), and references therein.

2 D. V. Averin and K. K. Likharev, in Mesoscopic Phenomena in Solids edited by B. L. Altshuler et al. (Elsevier, Amsterdam, 1991), p. 173, and references therein; apparently the first observations of the effect of the Coulomb blockade have been reported in I. Giaever and H. R. Zeller, Phys. Rev. Lett. 20, 1504 (1968) and J. Lambe and R. C. Jaklevic, Phys. Rev. Lett. 22, 1371 (1969).

${ }^{3}$ L. I. Glazman and K. A. Matveev, Zh. Eksp. Teor. Fiz., 98, 1834 (1990) [Sov. Phys. JETP, 71, 1031 (1990)].

${ }^{4}$ K. A. Matveev, Zh. Eksp. Teor. Fiz., 99, 1598 (1991) [Sov. Phys. JETP, 72, 892 (1991)].

${ }^{5}$ K. Flensberg, Phys. Rev. B 48, 11156 (1993).

${ }^{6}$ K. A. Matveev, Phys. Rev. B 51, 1743 (1995).

7 E. B. Kolomeisky, R. M. Konik, and X. Qi, Phys. Rev. B 66, 075318 (2002).

8 D. Berman, N. B. Zhitenev, R. C. Ashoori, and M. Shayegan, Phys. Rev. Lett. 82, 161 (1999).

9 E. B. Kolomeisky and J. P. Straley, Rev. Mod. Phys. 68, 175 (1996), and references therein.

10 S. - K. Ma, Modern Theory of Critical Phenomena, (Benjamin, Reading, MA, 1980), and references therein.

11 C. L. Kane and M. P. A. Fisher, Phys. Rev. B 46, 15233 (1992).

12 J. B. Kogut, Rev. Mod. Phys. 51, 659 (1979), and references therein.

13 The classical counterpart of the action (14) is implicit in R. E. Peierls, Helv. Phys. Acta 7, Suppl. II, 81 (1934); see also R. E. Peierls, in Surprises in Theoretical Physics (Princeton University Press, Princeton, NJ, 1979), Sec. 4.1.

14 L. D. Landau and E. M. Lifshitz, Statistical Physics, vol.V, Part 1, third edition, revised and enlarged by E. M. Lifshitz and L. P. Pitaevskii (Pergamon, 1980).

15 M. Abramowitz and I. A. Stegun, eds. Handbook of Mathe- matical Functions with Formulas, Graphs, and Mathematical Tables (Dover, New York, 1972, Section 5.2.

16 P. Nozières in Solids far from Equilibrium, edited by C. Godréche (Cambridge University Press, Cambridge, 1992), p. 1, and references therein; P. Nozières and F. Gallet, J. Physique, 48, 353 (1987).

17 A. Schmid, Phys. Rev. Lett. 51, 1506 (1983); F. Guinea, V. Hakim, and A. Muramatsu, ibid., 54, 263 (1985); M. P. A. Fisher and W. Zwerger, Phys. Rev. B 32, 6190 (1985); E. B. Kolomeisky, Pis'ma Zh. Eksp. Teor. Fiz. 47, 254 (1988) [JETP Lett. 47, 307 (1988); C. L. Kane and M. P. A. Fisher, Phys. Rev. Lett. 68, 1220 (1992); E. B. Kolomeisky and J. P. Straley, ibid., 76, 2930 (1996).

18 H. J. F. Knops and L. W. J. den Ouden, Physica, 103, 597 (1980).

19 R. E. Peierls, Quantum Theory of Solids (Oxford University Press, Oxford, 1955), Sec. 5.3; More Surprises in Theoretical Physics (Princeton University Press, Princeton, NJ 1991), Sec. 2.3.

20 E. B. Kolomeisky and J. P. Straley, Phys. Rev. B 53, 12553 (1996), and references therein.

21 The parameter $K$ used by Kane and Fisher ${ }^{11}$ is identical to $q^{2}$ of this paper.

22 D. J. Thouless, Phys. Rev. 187, 732 (1969).

23 Sec.163 of Landau and Lifshitz 14 .

24 J. M. Kosterlitz and D. J. Thouless, J. Phys. C 6, 1181 (1973); J. M. Kosterlitz, ibid. 7, 1046 (1974).

25 This can be arrived at by comparing Eqs.(4.2) of Kane and Fisher $^{11}$ with Eq. 10 of this work.

${ }^{26}$ P. W. Anderson and G. Yuval, J. Phys. C 4, 607 (1971); A. J. Bray amd M. A. Moore, Phys. Rev. Lett. 49, 1545 (1982); S. Chakravarty, ibid. 49, 681 (1982).

27 E. B. Kolomeisky, unpublished. 